\title{
Messungen des Intensitätssprungs an der Grenze der Balmer-Serie im Spektrum von B-, A- und F-Sternen
}

\author{
Von Gerhard Miczaika \\ Aus der Badischen Landessternwarte Heidelberg-Königstuhl \\ (Z. Naturforschg. 3 a, 129-134 [1947]; eingegangen am 15. November 1947)
}

\begin{abstract}
-Es werden Messungen des Intensitätssprungs im Kontinuum an der Grenze der BalmerSerie für 31 A-, B- und F-Sterne mitgeteilt und mit Messungen von B a r bi e r und $\mathrm{C}$ h a lo n g e verglichen. Die Abhängigkeit der Größe der Diskontinuität von Spektraltyp und absoluter Helligkeit für Zwerge, Riesen und Überriesen sowie für Sterne mit Emissionserscheinungen wird untersucht.
\end{abstract}

$\mathrm{D}$ ie spektralphotometrische Untersuchung der Intensitätsverteilung im ultravioletten Teil der Spektren von Sternen frühen Spektraltyps ist für die Theorie der Sternatmosphären in vieler Hinsicht von hervorragender Bedeutung. Die auffallendste Erscheinung im Kontinuum der B-, A- und F-Sterne zwischen $\lambda=3000$ und $\lambda=4000$ ist ohne $Z_{w}$ eifel der starke Intensitätssprung an der Grenze der Balmer-Serie bei $\lambda=3647$. Das $\mathrm{Zu}$ standekommen des Balmer-Sprungs hängt eng mit der Wellenlängenabhängigkeit des Absorptionskoeffizienten der Sternmaterie zusammen. Bezeichnet man den frequenzabhängigen Absorptionskoeffizienten mit $x_{v}$ und nimmt man Rücksicht darauf, daß es neben spontanen Emissionsprozessen solche gibt, die durch Strahlung erzwungen werden und deren Häufigkeit der Strahlungsdichte proportional ist, so hat man $x_{\nu}$ um das Verhältnis von erzwungener zu gesamter Emission zu verkleinern, erhält also

$$
x_{v}^{\prime}=x_{\nu}\left(1-e^{-\frac{h v}{k T}}\right) .
$$

Der Absorptionskoeffizient der Strahlung der Frequenz $\vee$ ist einfach definiert durch

$$
\frac{d J_{v}}{d s}=-x_{v} J_{v} .
$$

Für die Regelung der Gesamtstrahlung durch die Sternatmosphäre ist ein gewisser Mittelwert des Absorptionskoeffizienten (Rosselandsches Mittel)

$$
\frac{1}{\bar{x}} \frac{d J}{d s}=\int_{0}^{\infty} \frac{1}{x_{v}} \frac{d J_{v}}{d s} d v,
$$

bzw. bei Rücksichtnahme auf die erwähnte Kor- rektur

$$
\frac{1}{\bar{x}} \frac{d J}{d s}=\int_{0}^{\infty} \frac{1}{x_{v}^{\prime}} \frac{d J_{v}}{d s} d v
$$

verbindlich. Die Energieverteilung im Kontinuum wird durch $x_{y}^{\prime} / \bar{x}$ bestimmt. Der Strahlungsstrom ist durch die Temperatur $T_{0}$ der Hohlraumstrahlung der Intensität $J_{0}$ gegeben zu

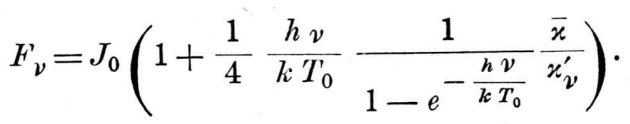

$x_{v}^{\prime} / \bar{x}$ stellt einen Mittelwert über alle Schichten des Sterns dar, die zur Ausstrahlung beitragen.

Auf der langwelligen Seite der Balmer-Grenze ist $x_{v}^{\prime} / \bar{x}<1$ für effektive Temperaturen von etwa $6000^{\circ}$ bis $15000^{\circ}$, so daß Strahlung aus tieferen Schichten hoher Temperatur nach außen dringen kann, die kontinuierliche Strahlung also stärker ist, als nach $T_{\boldsymbol{e}}$ zu erwarten wäre. Auf der kurzwelligen Seite dagegen ist $x_{v}^{\prime} / \bar{x}>1$, die Strahlung stammt überwiegend aus den äußersten kühlen Atmosphärenschichten, so daß $F_{v}$ nur noch wenig über $J_{0}$ liegt. Das Verhältnis der Größe des Strahlungsstroms zu beiden Seiten der Balmer-Grenze hängt zunächst einmal von der chemischen $\mathrm{Zu}$ sammensetzung der Sternatmosphäre, in erster Linie der relativen Häufigkeit von Metallen und Wasserstoff, dann aber auch von der effektiven Temperatur ab. Dies bedeutet gleichzeitig eine Abhängigkeit von Spektraltypus, absoluter Helligkeit oder Elektronendruck. Theoretische Rechnungen über diese Größe, die im folgenden durch

$$
D=\log \frac{F_{3647+\varepsilon}}{F_{3647-\varepsilon}^{\prime}}
$$


charakterisiert werden soll, haben vor allem $\mathrm{M}$ a c Crea ${ }^{1}$, Unsöld ${ }^{2}$, B a r bi e ${ }^{3}$ u. a. angestellt. Neuerdings haben $\mathrm{Ch}$ a $\mathrm{n} \mathrm{d}$ r a s e $\mathrm{k} h$ a r und $\mathrm{M} \ddot{\mathrm{u}} \mathrm{n} \mathrm{ch}^{4}$ die Größe der Diskontinuität unter Berücksichtigung des Beitrags, den die negativen Wasserstoffionen zum Absorptionskoeffizienten liefern, berechnet.

Spektralphotometrische Messungen sind in diesem Wellenlängengebiet noch nicht häufig ausgeführt worden. Die älteren Pionierarbeiten von C h ' I n g - S u n g Y ü ${ }^{5}$ und K a r p o v ${ }^{6}$ sind heute wegen der primitiven photometrischen Methoden, die sie benutzten, als überholt anzusehen; die Ergebnisse von $\mathrm{Y} \ddot{u}$ weichen in quantitativer Hinsicht auch systematisch von neueren ab. Messungen von Öhman ${ }^{7}$ sowie Öhman und Iwanowska ${ }^{8}$ beruhen auf Spektren kleiner Dispersion, die für statistische Untersuchungen erhalten worden waren. St r a s s ${ }^{9}$ hat einige Plejadensterne untersucht. Einige weitere Arbeiten betreffen nur wenige Sterne oder enthalten nur Relativmessungen.

Am ausführlichsten haben sich B a rbier, $\mathrm{Ch}$ a lo $\mathrm{g} \mathrm{g} \mathrm{e}^{10}$ und ihre Mitarbeiter mit der Energieverteilung zwischen $\lambda=3100$ und $\lambda=4600$ befaßt. Ihre Spektren wurden in Arosa (1900 m Seehöhe) bzw. auf der $3457 \mathrm{~m}$ hoch gelegenen Jungfraujoch-Station und der Sphinx $(3675 \mathrm{~m}) \mathrm{mit}$ einem Objektivprisma aus Quarz gewonnen. Die Höhenlage der Beobachtungsorte erleichtert das Arbeiten im UV, da hier die Extinktion der kurzwelligen Sternstrahlung durch die Erdatmosphäre sehr stark herabgesetzt ist. Das Programm umfaßte 204 helle Sterne, von denen die Größe des Balmer-Sprungs zum größten Teil bestimmt werden konnte.

Die vorliegende Arbeit hat die Ableitung des Intensitätssprungs für $31 \mathrm{~B}-$, A- und F-Sterne zum Ziel.

\section{Instrument}

Die Aufnahmen wurden mit dem neuen UV-Spektrographen von Zeiß, der im Newton-Fokus des Spiegel-

1 W. H. M a c C r e a, Monthly Notices Roy. Astronom. Soc. 91, 836 [1936].

${ }^{2}$ A. U n s öl d, Z. Astrophysik 8, 32 [1932]; 8, 225 [1934]; Physik der Sternatmosphären, S. 131, Berlin 1938.

3 D. B a r b i e r, Ann. d'Astrophysique 1, 317 [1938] ; 6, $113[1934]$.

4 S. C hand rase khar u. G. M ü n c h, Astrophysic. J. 104, 446 [1946].

5 C h'I n g - S u n g Y ü, Lick Observatory Bull. 12, 104 [1926]; 12, 155 [1926]; 15, 1 [1933]. [1934]. teleskops der Sternwarte Heidelberg angebracht ist, unter Mitwirkung von Hrn. U. B e cker erhalten. Das Spiegelteleskop hat eine Öffnung von $72 \mathrm{~cm}$ und eine Brennweite von $281 \mathrm{~cm}$. Zur vollen Ausnutzung der Reichweite des Spektrographen nach kurzen Wellenlängen hin ist eine Spiegelbelegung mit Aluminium erwünscht. Die Reflexionsfähigkeit eines Silberbelags sinkt unterhalb von $\lambda 4000$ sehr rasch, insbesondere wenn die Versilberung der Spiegeloberfläche älter wird. Die Spiegel trugen zur Zeit der hier benutzten Aufnahmen eine Silberbelegung, wodurch eine erhebliche Vergrößerung der Belichtungszeiten für den interessierenden Spektralbereich erforderlich wurde.

Der Spektrograph besitzt zwei $60^{\circ}$-Prismen aus UZK 5. Kollimator- und Kameraobjektive sind ebenfalls aus UV-Glas. Er kann sowohl als Spaltspektrograph wie auch spaltlos benutzt werden. Das positive Kollimatorobjektiv ist dreilinsig, besitzt $40 \mathrm{~mm}$ freie Öffnung und ist mit seinem Öffnungsverhältnis 1:3,9 dem des Spiegelteleskops angepaßt. Für die Aufnahmen stehen zwei Kameras zur Verfügung. Die eine ist mit einem Vierlinser vom Petzvaltyp von $50 \mathrm{~mm}$ freier Öffnung und $200 \mathrm{~mm}$ Brennweite, die andere mit einem Dreilinser vom Chromattyp von ebenfalls $50 \mathrm{~mm}$ freier Öffnung, aber $375 \mathrm{~mm}$ Brennweite ausgerüstet. Die Aufnahmen dieser Untersuchung wurden mit der kurzen Kamera ausgeführt. Zwischen dem letzten $60^{\circ}$ - Prisma und dem Kameraobjektiv kann ein Biprisma aus UV-Glas mit einem brechenden Winkel von $179^{\circ} 43^{\prime}$ so eingeschoben werden, daß das Spektrum der Länge nach in zwei nebeneinanderliegende aufgespalten werden kann, die je nach der Lage der brechenden Kante des Biprismas einen beliebig verschiedenen Anteil der zur Verfügung stehenden Gesamtintensität erhalten. Das eine Spektrum wird also gegenüber dem anderen durch Intensitäten erzeugt, die gegenüber den für das andere zur Wirkung kommenden um einen wellenlängenunabhängigen meßbaren Faktor verkleinert sind. Demselben $Z$ weck dient ein zweites Biprisma aus UV-Glas mit gleichem brechendem Winkel und einseitigem Platinbelag, das ein geschwächtes Nebenspektrum erzeugt, wobei natürlich durch Ändern der geometrischen Verhältnisse die Schwächung nicht variiert werden kann. Sie ist durch den Platinbelag gegeben und etwas wellenlängenabhängig.

Der Vorteil, auf diese Art Intensitätsstufen zu erzeugen, liegt auf der Hand. Die Intensitätsstufen entstehen unter völlig gleichen Bedingungen wie das zu

7 Y. Ö h m a n, Ark. Mat., Astron. Fysik 25 B Nr. 20 $=$ Stockholm Obs. Meddelande Nr. 30 [1937]; Stockholm Obs. Ann. 12, 1 [1935].

${ }^{8}$ Y. Öhman u. W. I w an ow ska, Ark. Mat., Astron. Fysik 25 B Nr. 7 = Stockholm Obs. Meddelande Nr. 21 [1935].

${ }^{9}$ H. Stras s l, Z. Astrophysik 5, 205 [1932].

10 A. A r nulf, D. B a rbier, D. Chalonge, R. Carnavaggi a, H. S a f ir u. E. Vassy, J. Physique Radium (VII) 6, 137 [1935] ; J. Observateurs 19, 149 [1936]; Ann. d'Astrophysique 1, 293 [1938]; Ann. d'Astrophysique 4, 30 [1941] u. a. O. 
photometrierende Spektrum. Insbesondere fällt ein Einfluß der Extinktion völlig fort.

Zur Verbreiterung der Sternspektren dient eine Planparallelplatte aus UBK 5, die im Lichtweg der Kamera kurz vor der Platte liegt und durch einen Synchronmotor um eine in der Dispersionsrichtung verlaufende Achse periodisch gekippt wird. Sie erlaubt Verbreiterungen von $0,08 \mathrm{~mm}$ bis $0,5 \mathrm{~mm}$.

Eine ausführliche Beschreibung des Spektrographen wird später an anderer Stelle gegeben werden.

\section{Die A f n a hmen}

Für die vorliegende Untersuchung wurden ausschließlich Perutz-Peromnia-Platten $4 \times 6 \mathrm{~cm}$ verwendet, die einheitlich $7 \mathrm{~min}$ in Geka-Rapid-Entwickler 1: 25 bei völliger Dunkelheit entwickelt wurden. Die Sterne wurden auf den Spaltbacken während der Exposition geführt und die Spektren mit der optischen Verbreiterungsvorrichtung auf $0,25 \mathrm{~mm}$ verbreitert. Die Breite des Spektrographenspaltes betrug im allgemeinen 0,055 bis $0,060 \mathrm{~mm}$, nur in einigen Fällen bei etwas stärkerer Bildunruhe wurde sie auf $0,070 \mathrm{~mm}$ vergrößert. Sämtliche Spektren wurden mit der Kamera von $200 \mathrm{~mm}$ Brennweite gewonnen. Die Dispersion betrug bei ihr in der Nähe des BalmerSprungs etwa $55 \AA / \mathrm{mm}$.

Im Strahlengang befand sich das Biprisma, dessen eine Hälfte mit Platin belegt war. Bei jeder Aufnahme entstanden also zwei nebeneinanderliegende Spektren, von denen das eine gegenüber dem anderen mit einem etwas wellenlängenabhängigen bekannten Bruchteil der Intensität des anderen belichtet worden war. Auf jeder Platte wurde derselbe Stern zweimal mit dazwischen erfolgendem Versetzen der Platte exponiert; sie trug also jeweils vier Spektren, die zur Auswertung kamen. Die Ermittlung der Absorption des Platinbelags in Abhängigkeit von der Wellenlänge wurde im Laboratorium ausgeführt. Intensitätsstufen wurden dabei durch Ändern der Entfernung Spektrographenspalt-Lichtquelle hergestellt. Die notwendigen Belichtungszeiten lagen für die Sterne dieses Programms zwischen 2 min und $60 \mathrm{~min}$.

Die Spektren sind im gesamten zur Abbildung kommenden Wellenlängenbereich scharf fokussiert. Die Balmer-Serie ist bei $\alpha$ Cyg bis zum 21. Glied erkennbar. Die Registrierung eines solchen Spektrums ( $\alpha$ Lyrae, AO) ist in Abb. 1 wiedergegeben.

\section{Registrierung der Spektren}

Die Registrierung der Spektren wurde mit dem lichtelektrischen Registrierphotometer von Zeiß auf Agfa-Registrierpapier vorgenommen. Sie erfolgte mit etwa 40-facher Úbersetzung für jede Platte in einem Arbeitsgang rasch hintereinander. Da nur ein kleines Stück des Spektrums für unsere Aufgabe interessierte, wurde die Registrierung auf den entsprechenden Teil - etwa von $\lambda 3550$ bis $\lambda 3900$ - beschränkt. Versuche erwiesen die Durchregistrierung des Plattenuntergrundes längs des Spektrums als unnötig. Die Richtung, in der registriert wurde, war für alle Spektren dieselbe, um eventuelle unterschiedliche Einflüsse durch Trägheit des Photometers zu vermeiden. Die Breite des Lichtbündels, das durch die Platte gegangen war, wurde vor dem Auftreffen auf die Zelle auf 0,05 bis $0,15 \mathrm{~mm}$ begrenzt. Die Spalthöhe wurde etwas geringer gemacht als die Höhe der Spektren senkrecht zur Dispersionsrichtung. Registriert wurden also nicht Bildstärken, sondern echte Schwärzungen. Es wurde sorgfältig darauf geachtet, daß die Registrierungen genau in der Richtung der Längsachse der Spektren erfolgten und diese unter der Mitte des photometrierenden Lichtbündels lag. Die Lampenspannung bestimmte sich, wie in geringem Umfang auch Spalthöhe und Spaltbreite, durch den auf der Platte zu registrierenden Schwärzungsbereich und das Bestreben, die Kornschwankungen möglichst

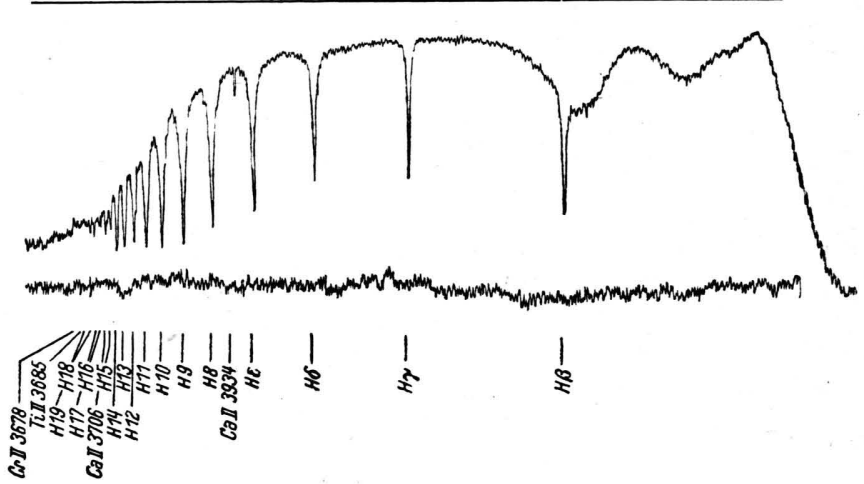

Abb. 1. Registrierung des Spektrums von $\alpha$ Lyrae (Spektraltypus AO).

weit herabzudrücken. Während der Registrierung einer Platte wurde der Zustand des Photometers völlig konstant gehalten. Die Registriergeschwindigkeit wurde ebenfalls einheitlich eingestellt. Jedes Registrierblatt trug die Marken für "Klare Platte“ und ,Völlige Dunkelheit“.

Die Schwärzungskurven wurden in bekannter Weise ausgeführt, die dazu notwendige Ausmessung der Registrierkurven geschah mit einem auf Glas geritzten Maßstab; 0,1 mm wurden geschätzt. Die erreichbare Genauigkeit entsprach durchaus den Anforderungen, da $1 \mathrm{~mm}$ Ordinate etwa $0^{m}, 08$ darstellt. Da die Schwärzungskurven nur etwa über eine Größenklasse benötigt wurden, konnten sie unbedenklich aus einem etwa $400 \AA$ d breiten Bereich abgeleitet werden. Eine Wellenlängenabhängigkeit ist in einem so schmalen Spektralbereich kaum zu befürchten bzw. spielt bei dem kleinen hier in Betracht kommenden Intensitätsintervall keine Rolle. Praktisch sind die Schwärzungskurven gerade Linien. Die Festlegung wellenlängenmäßig zusammengehöriger Punkte konnte mit dem Glasmaßstab durch Anschluß an die Wasserstofflinien genügend genau vorgenommen werden.

Eine gewisse Schwierigkeit bei der Ermittlung des Kontinuums bilden die durch die Körnigkeit der Platte hervorgerufenen zickzackförmigen Unregel- 


\begin{tabular}{|c|c|c|c|c|c|c|c|}
\hline Stern & $m_{v}$ & Sp. & $D$ & Stern & $m_{v}$ & Sp. & $D$ \\
\hline$\alpha \mathrm{An}$ & 2,15 & $0 p$ & 0,240 & $\gamma \mathrm{Cyg}$ & 2,32 & $\mathrm{cF} 8$ & 0,144 \\
\hline$\alpha \mathrm{Aqr}$ & 3,19 & Go & 0,0 & $41 \mathrm{C}$ & & cF5 & \\
\hline$a \mathrm{Aql}$ & 0,89 & A 5 & 0,3 & $a \mathrm{De} 1$ & 3, & B 8 & 76 \\
\hline$\delta \mathrm{Aql}$ & 3,44 & Fo & 0,2 & $\zeta$ Dra & 3, & B5 & \\
\hline$\zeta \mathrm{Aql}$ & 3,02 & $\mathrm{~A} 0$ & 0,4 & * Dra & 3,8 & $\mathrm{~B} 5 \mathrm{e}$ & 0,248 \\
\hline$\lambda \mathrm{Aql}$ & 3,55 & B9 & 0,4 & $a \mathrm{Lyr}$ & 0,1 & A 0 & 0,476 \\
\hline$\beta$ Ari & 2,72 & A 5 & 0,4 & $\gamma \mathrm{Lyr}$ & 3,3 & $\mathrm{~A} 0 \mathrm{p}$ & 0,360 \\
\hline$\beta$ Cas & 2,42 & dF5 & 0,2 & a Peg & 2,5 & $\mathrm{AO}$ & 0,456 \\
\hline$\gamma 0$ & var. & $\mathrm{BOe}$ & $-0,0$ & $\gamma \mathrm{Peg}$ & 2,8 & B 2 & 0,148 \\
\hline & 2,8 & A5 & 0,4 & $\zeta$ Peg & 3,6 & B 8 & 0,384 \\
\hline & 3,4 & B 3 & 0,2 & i $\mathrm{Peg}$ & 3,7 & A 2 & 0,392 \\
\hline$\zeta \mathrm{C}$ & 3,72 & B 3 & 0,1 & $a \mathrm{Per}$ & 1, & cF5 & 0,328 \\
\hline & 4,24 & $\mathrm{cBOe}$ & 0,060 & $v$ Per & 3 & c F5 & 0,240 \\
\hline$\alpha$ Cep & 2,60 & A 5 & 0,380 & $\beta$ Tri & 3,0 & A 5 & 0,431 \\
\hline$\beta$ Cep & 3,33 & B 1 & 0,040 & $a \mathrm{UMi}$ & 2,12 & $\mathrm{cF} 8$ & 0,260 \\
\hline$\alpha$ Cyg & 1,33 & $\mathrm{cA} 2 \mathrm{e}$ & 0,388 & & & & \\
\hline
\end{tabular}

Tab. 1. Gemessene Intensitätssprünge.

mäßigkeiten der Registrierkurve. Mit später werdendem Spektraltyp macht sich auch der steigende Einfluß der zunehmenden Linienzahl bemerkbar. Bei den B- und A-Sternen wird die Festlegung des Kontinuums durch die Überlagerung der höheren BalmerLinien in der Nähe der Seriengrenze beeinflußt. Die Festlegung des Kontinuums auf den Registrierkurven ist in erheblichem Umfang Sache der persönlichen Auffassung. Ich bin ähnlich wie Stra B $1^{\mathbf{1 1}}$ verfahren und habe einen etwa $1,5 \mathrm{~mm}$ breiten Rand gegenüber der Registrierung ,Völlige Dunkelheit“ gemessen, dabei aber die äußersten Zacken, die offensichtlich als Störungen anzusehen sind, unberücksichtigt gelassen. Entscheidend ist, daß Einheitlichkeit der Auffassung gewahrt bleibt.

\section{Ergebnisse}

Um die Ergebnisse mit denen von B a r bie r und Chalonge aus ihrer letzten zusammenfassenden Arbeit ${ }^{12}$ vergleichbar zu machen, wurde die Sprunghöhe bei $\lambda=3700$ gemessen. Die Stelle ist leicht als Mitte zwischen den Balmer-Linien H 16 und H 17 zu erkennen. Tab. 1 gibt die Werte für den Betrag des Intensitätssprungs $D$ wieder, durch Multiplikation mit 2,5 erhält man diesen Unterschied in Größenklassen. Angegeben sind ferner die scheinbare visuelle Helligkeit $m_{v}$ und der Spektraltyp nach dem Henry-Draper-Katalog.

Sterne meines Programms sind auch von B a r bier und $\mathrm{Chal}$ onge gemessen worden. In Abb. 2 sind zum Vergleich zusammengehörige Werte gegeneinander aufgetragen. Wie aus der Lage der Punkte zur $45^{\circ}$ - Geraden ersichtlich ist,

11 H. Str a ß l, Z. Astrophysik 5, 205 [1932].

12 D. B arbier u. D. Chalonge, Ann. d'Astrophysique 4, 30 [1941]. kann von verbürgbaren systematischen Abweichungen nicht gesprochen werden. Die mittlere gegenseitige Abweichung beträgt bei den gemeinsamen Sternen $\pm 0,03$, ist also durchaus befriedigend. Ein Vergleich mit den alten Messungen von $\mathrm{Yü}$ und Karpov ist bereits von Arnulf, $\mathrm{B}$ a r bi er und $\mathrm{Ch}$ a $\mathrm{l}$ on $\mathrm{g} \mathrm{e}^{\mathbf{1 3}}$ vorgenommen wor-

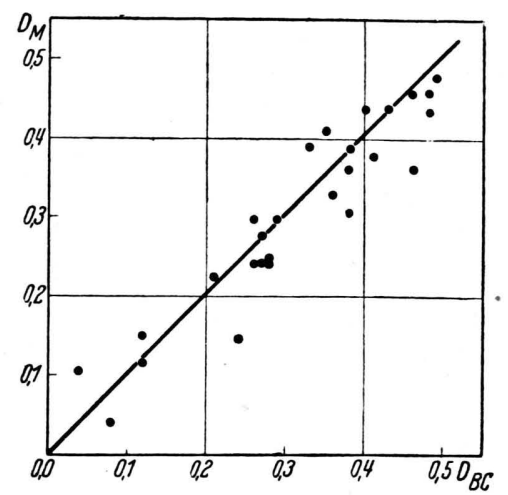

Abb. 2. Vergleich der $D$-Werte von $\mathrm{M}$ i c z a i k a und $\mathrm{B}$ a r b i e r - Ch a long e.

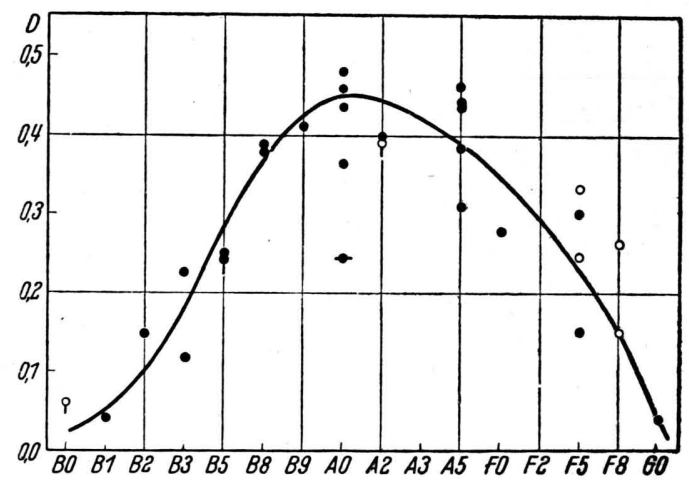

Abb. 3. Abhängigkeit des Balmer-Sprungs vom Spektraltyp. o Überriesen, i Sterne mit Emissionscharakteristik, - $-\alpha$ Andromedae.

den und erübrigt sich daher hier, zumal die Zahl der gemeinsamen Sterne auch nur gering ist.

In Abb. 3 sind die gemessenen Werte des Intensitätssprungs gegen den Spektraltyp der Sterne aufgetragen. Die Sterne mit $c$-Charakteristik sind besonders gekennzeichnet, ebenso solche mit Emissionserscheinungen. Die Feststellung von $\mathrm{B}$ a r bier und Chalonge, daß die Überriesen mit Spektren früher als A 5 einen kleineren und die Überriesen mit Spektren später als A 5 einen grö-

13 A. Arnulf, D. B a r bier u. D. Chalonge, J. Observateurs 19, 149 [1936]. 
ßeren Intensitätssprung an der Balmer-Grenze als die gewöhnlichen Sterne aufweisen, geht aus unserem Material nicht sicher hervor, wenn auch Andeutungen dafür erkennbar sind.

Erheblich heraus fällt $\propto$ Andromëdae. Sein Spektrum wird im Henry-Draper-Katalog nach den Wasserstoff- und Calciumlinien zu A 0 klassifiziert, nach der Stärke von $\lambda=4062,2, \lambda=4267,4$ und $\lambda=4471,5$ entspricht es aber eher B 8 . M organ ${ }^{14}$ macht ebenfalls darauf aufmerksam, daß die HeI -Linien sowie $\lambda=4267$ (C II), einige Fe II-, Ti II-, Si II - Linien und $K$ (CaII) eher für B 8 sprechen. Temperatur und Größe des BalmerSprungs legen nach Barbier und Chalonge ${ }^{12}$ sogar B 5 nahe. Der hier gefundene Betrag für den Balmer-Sprung führt ebenfalls auf B 5. Die absolute Helligkeit ist nach der Parallaxe $-0^{m}, 2$, paßt also auch besser zu B 8 bis B 5 als zu A 0 . Bei einigen Sternen des Programmes treten Emissionslinien auf; abgesehen von $\gamma$ Cassiopeiae sind diese schwach, $\gamma$ Cassiopeiae wurde mit Rücksicht auf die Veränderlichkeit seines Spektrums bei der weiteren Diskussion ausgeschlossen. Die übrigen $\boldsymbol{e}$-Sterne fügen sich in den allgemeinen Kurvenverlauf ein.

Um das Verhalten der Überriesen in bezug auf den Balmer-Sprung zu untersuchen, ist das hier mitgeteilte Material zu klein. Unter Heranziehung der Messungen von $\mathrm{B}$ a r bier und $\mathrm{Ch}$ a l o n ge - bei gemeinsamen Programmsternen wurden Mittel gebildet - zeigt sich zunächst, daß die Diskontinuität ihr Maximum nicht mehr bei A 0 , sondern bei etwa F 0 hat. Der Abfall nach B 0 ist daher erheblich flacher als nach G0. Dieses Verhalten ist $\mathrm{zu}$ erwarten. Der Elektronendruck ist in ihren Atmosphären sehr viel kleiner als in den normalen Sternen, und die Absorption durch Wasserstoff stärker gegenüber der durch die Metalle.

Die Abhängigkeit der Größe des BalmerSprungs von der absoluten Helligkeit ist in Abb.5 für Riesen und Zwerge gemeinsam dargestellt. Das Material stammt aus der Untersuchung von $\mathrm{B}$ a r b i e r und $\mathrm{Ch}$ al o n $\mathrm{ge}^{12}$ und der vorliegenden. Zur Berechnung der absoluten Helligkeiten dienten die Parallaxen aus dem Katalog von Schlesinger ${ }^{15}$. Sterne mit $c$-Charakteristik - Überriesen - sind fortgelassen, dagegen wur-

14 W. W. M o r g a n, Astrophysic. J. 77, 330 [1933].

15 F.S c h l e sing e r, General Catalogue of Stellar Parallaxes, New Haven 1935. den Sterne mit Emissionserscheinungen besonders markiert eingezeichnet. Es handelt sich dabei um Sterne mit weit ausgedehnten Hüllen, die sich nicht im lokalen thermodynamischen Gleichgewicht befinden; teilweise dürften sich darunter auch noch Überriesen befinden. Oft besitzen nur $\mathrm{H} \alpha$ oder einige andere Linien Emissionskontu-

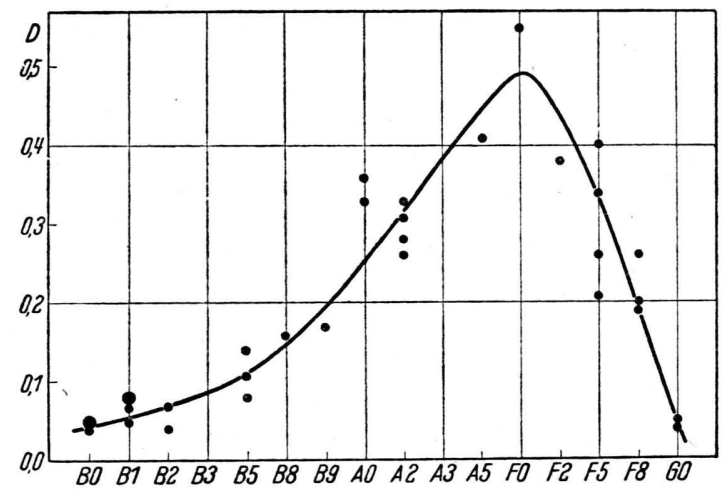

Abb. 4. Abhängigkeit des Balmer-Sprungs vom Spektraltyp bei Überriesen.

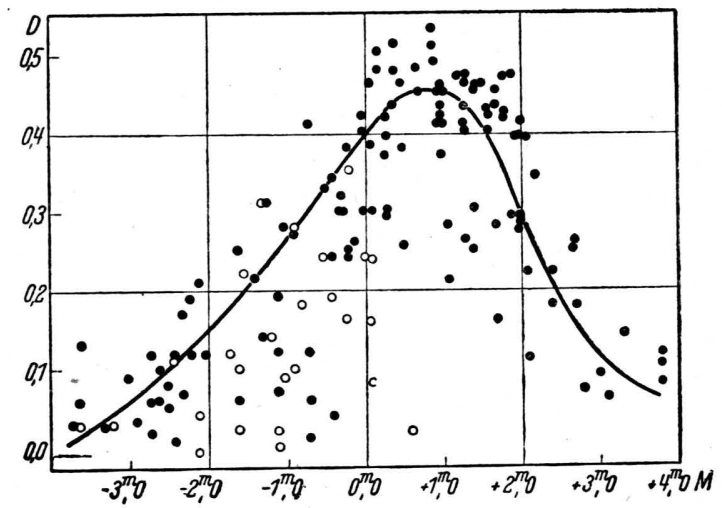

Abb. 5. Abhängigkeit des Balmer-Sprungs von der absoluten Helligkeit (Riesen und Zwerge). o Sterne mit Emissionscharakteristik.

ren; gelegentlich erscheint aber auch das Kontinuum, das sich an die Balmer-Grenze anschließt, in Emission, wodurch es unter Umständen zu einer Umkehrung des Intensitätsverhältnisses zwischen beiden Seiten der Seriengrenze kommen kann. Es ist also zu erwarten, daß Sterne frühen Spektraltyps mit Emissionserscheinungen durch Überlagerung einer kontinuierlichen Emission zumindest eine Neigung zu kleineren $D$-Werten, als ihrem Spektraltyp entspricht, aufweisen werden. Diese Sterne gehören überwiegend zum Typ B, häufen sich also wegen ihrer großen absoluten 
Helligkeit in der linken unteren Ecke der Abb. 5 . Sie zeigen tatsächlich die Tendenz zu kleineren $D$-Werten, als ihrer absoluten Helligkeit nach zu erwarten wäre. Der Verlauf der Kurve ist ohne Rücksicht auf sie gezogen. Das Maximum des Balmer-Sprungs wird bei $\mathrm{M}=+1^{m}, 0$ erreicht, entsprechend dem Spektraltyp A 0 .

Die absoluten Helligkeiten der Überriesen sind zu unsicher, um die Abhängigkeit der $D$-Werte von ihnen betrachten zu können. Vermutlich liegen sie überwiegend auf einem Ast, der in der Abb. 5 parallel $\mathrm{zu}$ dem von links ansteigenden verläuft, aber um etwa $2^{m}$ zu größeren Helligkeiten verschoben ist. Die Verhältnisse in den Atmosphären weichen von denen der normalen Riesen und Zwerge infolge des stark verringerten Druckes erheblich ab. Der Anteil am Absorptionskoeffizienten durch Streuung an freien Elektronen, dem bei den Riesen und Zwergen keine Bedeutung zukommt, führt zu einer merklichen Veränderung ihrer atmosphärischen Verhältnisse gegenüber diesen.

\title{
Zur Theorie der Streuung langsamer Neutronen an freien Protonen
}

\author{
Von ERICH HÜCKel \\ Aus der theoretischen Abteilung des Physikalischen Instituts der Universität Marburg \\ (Z. Naturforschg. 3 a, 134-142 [1948]; eingegangen am 8. Dezember 1947)
}

\begin{abstract}
Zur Berechnung des Streuquerschnitts von Protonen gegenüber langsamen Neutronen wird der Ansatz eines spinabhängigen Potentials der Form $-A \cdot \exp (-r / a)$ zugrunde gelegt. Mit den Werten $a=2,18 \cdot 10^{-13} \mathrm{~cm}, A_{T}=32,1 \mathrm{MeV}$ für die ${ }^{3} S$-Kraft und $A_{S}=10,2 \mathrm{MeV}$ für die ${ }^{1} S$-Kraft, welcher Wert einem virtuellen ${ }^{1} S$-Zustand entspricht, erhält man gute Übereinstimmung mit den neuen experimentellen Werten für den Streuquerschnitt im Energiebereich 0,035 bis etwa $2 \mathrm{MeV}$. Das Ergebnis spricht gegen die Existenz eines reellen $1 S$-Zustandes, wenn auch hieraus allein nicht eindẹutig zwischen den beiden Möglichkeiten: virtueller oder reeller ${ }^{1}$ S-Zustand, entschieden werden kann. Als Grenzwert des Streuquerschnitts für verschwindende Neutronenenergie erhält man etwa 20,6 $\cdot 10^{-24} \mathrm{~cm}^{2}$. Es wird ferner die Rechnung für einen ,,rechteckigen Potentialtopf" durchgeführt und nachgewiesen, daß die hierfür allgemein in der Literatur zu findende Formel unrichtig ist. Die richtige Formel wird angegeben. Man erhält einen virtuellen ${ }^{1} \mathrm{~S}$-Zustand mit einer Energie von der Größenordnung 1,5 MeV im Gegensatz zu den bisher angegebenen Werten, die zwischen etwa 60 und $120 \mathrm{KeV}$ liegen sollten. Bei richtiger Rechnung ergibt sich hier, wenn der $1 S$-Zustand virtuell ist, ein Verhältnis der ${ }^{3} S$ - zur $1 S$-Kraft etwa wie $3: 2$.
\end{abstract}

$\mathrm{D}_{\mathrm{s}}^{\mathrm{i}}$ ie Streuung von Neutronen an Protonen ist schon früh theoretisch behandelt worden ${ }^{1-6}$, doch sind exakte Messungen des Streuquerschnitts für Neutronen wohldefinierter Energie, die einen genauen Vergleich mit den theoretischen Ergebnissen ermöglichen, erst in jüngster Zeit von amerikanischen Autoren vorgenommen worden.

Es sind dies Messungen von Bailey und Mitarbb. ${ }^{7}$ über die Streuung monochromatischer Neutronen an Graphit und Cyclohexan im Ener-

1 E. W i g n e r, Z. Physik 83, 253 [1933].

${ }^{2}$ H. A. B e th e u. R. P e i e rs, Proc. Roy. Soc. [London] Ser. A 149, 176 [1935].

${ }^{3}$ E. F e enberg u. J. K. K n i pp, Physic. Rev. 48, 906 [1935].

${ }_{4}$ H. A. B e the u. R. F. B a che r, Rev. Mod. Physics 8, 114 [1936].

${ }^{5}$ H. D o l c h, Z. Physik 100, 401 [1936]. giebereich von 0,35 bis $6 \mathrm{MeV}$, von $\mathrm{Fr}$ is ch${ }^{8}$ mit Neutronen aus ${ }^{7} \mathrm{Li}(\mathrm{p}, \mathrm{n})$ zwischen 0,035 und $0,49 \mathrm{MeV}$ sowie von $\mathrm{C}$ o on und $\mathrm{Barschal1}{ }^{9}$ mit Neutronen von $2,5 \mathrm{MeV}$.

Diese Messungen lassen es möglich erscheinen, genauere Aussagen über die zwischen Proton und Neutron wirkenden Kräfte zu gewinnen.

In dem betrachteten Energiebereich ist einerseits die Energie der Neutronen groß gegen die chemische Bindungsenergie der Protonen, so daß

${ }^{6}$ C. K itte l u. G. B r e it, Physic. Rev. 56, 744 [1939].

7 B ailey, Benett, B ergstralh, Nuckoles, Richards u. Willi ams, Physic. Rev. 70, 583 [1946].

8 D. H. F r i s c h, Physic. Rev. 70, 589 [1946].

9 Coon u. Barschall, Physic. Rev. 70, 592 [1946]. 